\title{
Optical solitons of conformable space-time fractional NLSE with Spatio-temporal dispersion
}

\author{
Elif Yaşar and Emrullah Yaşar \\ Department of Mathematics, Faculty of Arts and Sciences, Bursa Uludag University, Bursa, Turkey
}

Received: 5 February 2018, Accepted: 18 July 2018

Published online: 7 August 2018.

\begin{abstract}
In this work, the dynamics of traveling waves of an improved nonlinear space-time fractional Schrödinger's equation with spatio-temporal dispersion in addition to group-velocity dispersion are studied. This equation models the propagation of solitons through nano optical fibers. The fractional derivative form is considered in the meaning of conformable fractional derivative. The $\exp (-\phi(\xi))$ algorithm will be carried out for retrieving the optical soliton solutions containing the form of kink and multi-soliton shapes. In addition, some graphical simulations of solutions are provided for better understanding the physical phenomena.
\end{abstract}

Keywords: Solitons, conformable fractional derivative, Spatio-temporal dispersion, $\exp (-\phi(\xi))$ method.

\section{Introduction}

The research area of optical soliton propagation is a quite important in nonlinear optics. This special type wave propagation can be observed in many physical models. For example, optical solitons form the basic carriers for signal propagation across long distances. Thanks to several advances in this field, many fascinating developments have placed in our daily lives such as internet activity and social media [1]. In the last two decades, the optical solitons have been examined from different aspects such as birefringent fibers, polarization mode dispersion, conservation laws, etc. [2]. In the open literature, many powerful methods have been developed for nonlinear evolution equations in the meaning of integrability. Some of them are listed in $[3,4,5,6,7,8,9,10]$.

The governing mathematical equation of optical solitons is nonlinear Schrödinger equation (NLSE)

$$
i q_{t}+a q_{t x}+b q_{x x}+c F\left(|q|^{2}\right) q=0, \quad i=\sqrt{-1}
$$

As can be seen, the above equation contains the nonlinear term and the group velocity dispersion (GVD), besides the linear evolution term. Therefore, the temporal optical soliton formation emerges delicate balance between GVD and nonlinearity. These type optical solitons propagate along trans-continental distances along the optical fibers [2].

However, as particularly emphasized in [11,12], the Eq.(1) is an ill-posed model. Therefore, the spatio-temporal dispersion (STD) besides to GVD and nonlinear terms is added to make the model well-posed.

On the other hand, a physical phenomenon may depend not only on the time (or space) instant but also on the time (or space) history, which can be successfully modelled using the theory of derivatives and integrals of fractional order [13, 14]. This interesting topic goes back to L'Hospital's famous question in 1695 asked what does it mean $\frac{d^{n} f}{d x^{n}}$ if $n=\frac{1}{2}$. We observe several approaches to the generalization of the notion of differentiation of fractional orders, e.g., 
Riemann-Liouville, Grünwald-Letnikow, Caputo and modified Riemann-Liouville derivative [15,16,17,18], etc.

In this paper, our aim is to build optical soliton solutions for improved space-time fractional NLSE with spatio-temporal dispersion

$$
i D_{t}^{\alpha} q+a D_{t}^{\alpha} D_{x}^{\alpha} q+b D_{x}^{\alpha} D_{x}^{\alpha} q+c F\left(|q|^{2}\right) q=0, \quad i=\sqrt{-1}
$$

in the sense of conformable fractional derivative. This new type fractional derivative is introduced by Khalil et al. [19] in 2014 and some setbacks of other definitions are improved.

We observe several interesting works on the Eq.(2) including integer order derivatives. Savescu et. al. constructed optical solitons for cases of Kerr law, power law, parabolic law, dual-power law as well as the log law via ansatz method [22]. Bhrawy et al. added to those results the cases of polynomial and triple-power laws by using the travelling wave hypothesis [2]. Very recently, Yıldırım et al. constructed systematically dark, bright and singular soliton solutions of model equation by extended Kudryashov's algorithm [23]. In addition to those papers, Biswas et al. obtained the dark, singular and singular periodic solutions employing by trial equation method [24].

The main motivation of this work is to examine the Eq.(2) via $\exp (-\phi(\xi))$ approach. As noted in the above lines, studying the Eq.(2) in the context of fractional derivative makes the physical model much more realistic. The three types of nonlinearity -Kerr law, parabolic and dual power law-associated with Eq.(2) will be considered.

The paper is structured thusly. In Section 2, conformable fractional derivate and $\exp (-\phi(\xi))$ method for handling the reduced ordinary differential equations (ODEs) are presented. Section 3 is devoted to present the optical soliton solutions for three distinct nonlinear cases. In the final section, we give some concluding remarks.

\section{Succinct overview of the conformable fractional derivative operator}

\subsection{Conformable fractional derivative operator}

In 2014, Khalil et.al [19] have developed a quite important and efficient new fractional derivative calling as the conformable fractional derivative operator. Its efficiency can be observed especially in fractional differential equations in both directions -i.e. space and time regard- containing this type new derivative operator.

Now we give the brief definition and its important properties which will be employed in subsequent sections for constructing the traveling wave solutions of improved space-time fractional NLSE with spatio-temporal dispersion.

Let $f:[0, \infty) \rightarrow \mathbb{R}$ be a function. The $\alpha$ th order conformable fractional derivative of $f$ is given by

$$
D^{\alpha}(f)(t)=\lim _{\varepsilon \rightarrow 0} \frac{f\left(t+\varepsilon t^{1-\alpha}\right)-f(t)}{\varepsilon}
$$

where $t>0$ and $\alpha \in(0,1]$.

In what follows, we give some important properties of above fractional derivative operator without proofs [19].

(1) $D^{\alpha}(c f+d g)=c D^{\alpha}(f)+d D^{\alpha}(g)$ for all $c, d \in \mathbb{R}$.

(2) $D^{\alpha}\left(t^{p}\right)=p t^{p-\alpha}$, for all $p \in \mathbb{R}$.

(3) $D^{\alpha}(\lambda)=0$ for all constant functions $f(t)=\lambda$.

(4) $D^{\alpha}(f g)=f D^{\alpha}(g)+g D^{\alpha}(f)$. 
(5) $D^{\alpha}\left(\frac{f}{g}\right)=\frac{g D^{\alpha}(f)-f D^{\alpha}(g)}{g^{2}}$.

(6) $D^{\alpha}(f \circ g)(t)=t^{1-\alpha} g^{\prime}(t) f^{\prime}(g(t))$.

\section{$2.2 \operatorname{Exp}(-\phi(\xi))$ method}

The $\exp (-\phi(\xi))$ method $[20,21]$ is one of the powerful approach for handling the nonlinear fractional differential equations. As can be seen below, there exist many solution sets according to coefficient classifications of the auxiliary equation. In the literature, we observe many studies related with this algorithm. For instance,in [25] the authors adopted the $\exp (-\phi(\xi))$ method to construct the solitary wave solutions of the fractional generalized Sawada-Kotera equation. Hosseini et. al. yielded the exact solutions of density-dependent conformable space-time fractional diffusion-reaction equation using the $\exp (-\phi(\xi))$ method [26].

Let's consider the space-time conformable fractional polynomial complex evolution equations as follows:

$$
P\left(q, D_{t}^{\alpha} q, D_{x}^{\alpha} q, D_{x}^{\alpha} D_{x}^{\alpha} q, D_{t}^{\alpha} D_{x}^{\alpha} q, \cdots\right)=0
$$

We give the main steps of this method as follows:

Step 1. We define the wave variable transformation

$$
q(x, t)=U(\xi) e^{i \Phi(x, t)},
$$

where $U(\xi)$ represents the shape of the pulse and

$$
\begin{gathered}
\xi=\frac{x^{\alpha}}{\alpha}-v \frac{t^{\alpha}}{\alpha}, \\
\Phi(x, t)=-\kappa \frac{x^{\alpha}}{\alpha}+w \frac{t^{\alpha}}{\alpha}+\theta .
\end{gathered}
$$

The function $\Phi(x, t)$ is the phase component of the soliton, $\kappa$ is the soliton frequency, $\omega$ is the wave number of the soliton, $\theta$ is the phase constant and $v$ is the velocity of the soliton. If one uses the variable transformation (4) through (5) in Eq.(3), then original equation Eq.(3) converts to

$$
E\left(U, U^{\prime}, U^{\prime \prime}, \ldots\right)=0
$$

nonlinear integer order ordinary differential equation (ODE).

Step 2. To gain the solution(s) of Eq.(6), we suppose that the form of solutions can be in finite series form

$$
U(\xi)=\sum_{i=0}^{N} a_{i} \exp (-\phi(\xi))^{i}=a_{0}+a_{1} \exp (-\phi(\xi))+\ldots+a_{N} \exp (-N \phi(\xi)), \quad a_{N} \neq 0
$$

where the arbitrary constants $a_{n}, n=0,1,2, \ldots, N$ should be fixed via the pursued algorithm, $N$ is a positive number, which is determined by the highest order linear term and nonlinear terms. In this stage, we assume that the $\phi(\xi)$ is a solution of the following auxiliary ODE:

$$
\phi^{\prime}(\xi)=\exp (-\phi(\xi))+\mu \exp (\phi(\xi))+\lambda
$$


According to coefficient classifications of Eq.(8), we have the following solution sets:

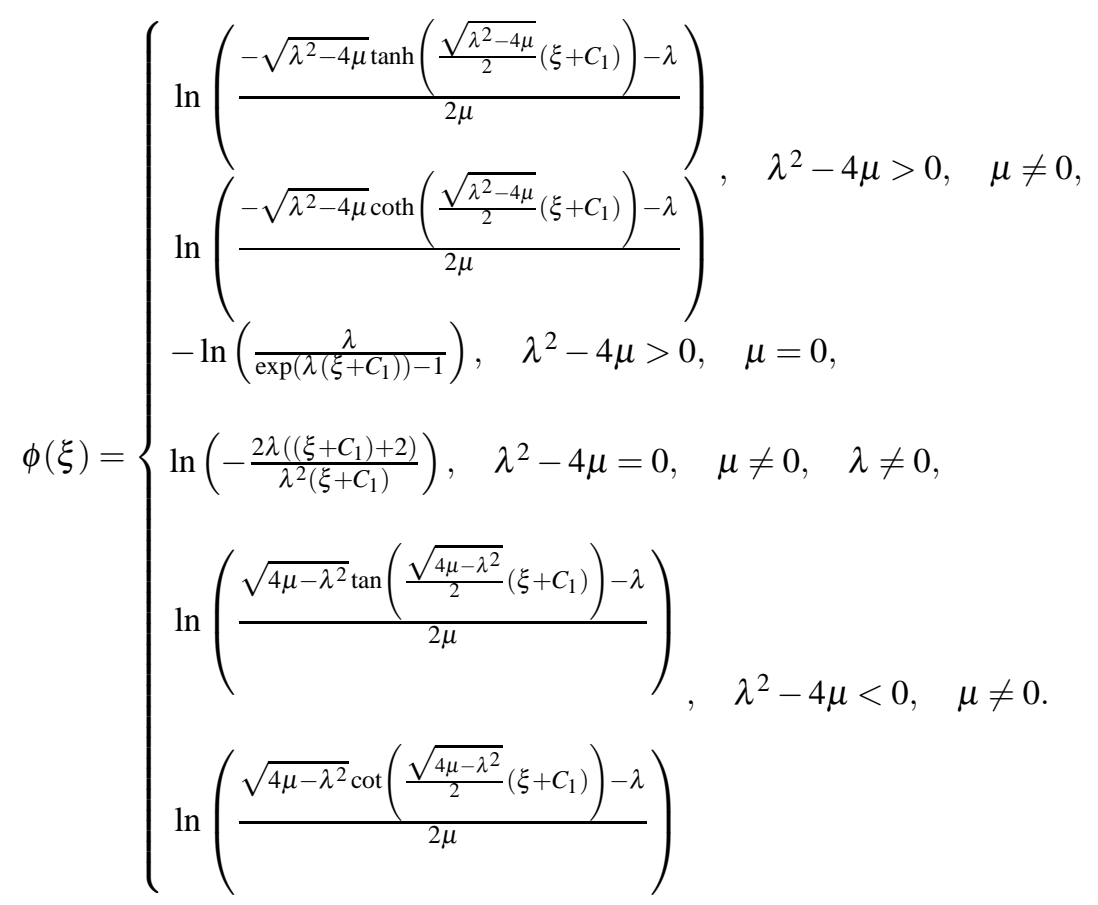

where $a_{n}, \cdots, \lambda, \mu$ are constants to be determined later.

Step 3. Plugging Eq.(7) into Eq.(6) with the aid of Maple and putting the coefficients of same powers of $\exp (-\phi(\xi))$ to zero, we obtain an algebraic system for getting $a_{n}$ 's.

Step 4. Substituting these coefficients values and the solutions of Eq.(8) into Eq.(6) we obtain the exact solutions of Eq.(3).

\section{Conformable fractional space-time NLSE with Spatio-temporal dispersion model and exact traveling wave solutions}

In this section, our purpose is to reveal the optical solitons of the improved NLSE with spatio-temporal dispersion which is defined in the form of space-time conformable fractional derivative. First, we present mathematical background of the model and then application of the $\exp (-\phi(\xi))$ method to the model equation.

\subsection{Model equation}

The dimensionless form of NLSE is given by ([24])

$$
i D_{t}^{\alpha} q+a D_{t}^{\alpha} D_{x}^{\alpha} q+b D_{x}^{\alpha} D_{x}^{\alpha} q+c F\left(|q|^{2}\right) q=0, \quad i=\sqrt{-1}
$$

where $x$ represents the non-dimensional distance along the fiber, $t$ represents temporal variable in dimensionless form and $a, b$ and $c$ are real valued constants. The dependent variable $q(x, t)$ is a complex valued wave profile.

The coefficient of the constant $a$ is spatio-temporal dispersion and the coefficient of constant $b$ is group-velocity 
dispersion. In addition, $c$ is the coefficient of the nonlinear term where the functional $F$ represents the non-Kerr law nonlinearity in general. The nonlinear function $F\left(|q|^{2}\right) q$ is $k$ times continuously differentiable, so that ([24])

$$
F\left(|q|^{2}\right) q \in \bigcup_{m, n=1}^{\infty} C^{k}\left((-n, n) \times(-m, m) ; \quad \mathbb{R}^{2}\right) .
$$

Plugging the Eq.(4) into Eq.(10) and then decomposing into imaginary and real parts, the following equations are deduced

$$
\begin{gathered}
v=\frac{a \omega-2 b \kappa}{1-a \kappa}, \\
(b-a v) U^{\prime \prime}-\left(w-a w \kappa+b \kappa^{2}\right) U+c F\left(U^{2}\right) U=0,
\end{gathered}
$$

respectively, where integration constants is chosen as zero in each integration process.

\subsection{Application of the $\exp (-\phi(\xi))$ method to space-time NLSE with spatio-temporal dispersion}

In this sub-section, the exact solutions of conformable improved space-time fractional NLSE with spatio-temporal dispersion will be retrieved by using the $\exp (-\phi(\xi))$-expansion scheme.

\section{Kerr-law nonlinearity}

The Kerr law of nonlinearity originates from the fact that a light wave in an optical fiber faces nonlinear responses from non-harmonic motion of electrons bound in molecules, caused by an external electric field [24].

The Kerr law nonlinearity is the case when $F(s)=s$. For Kerr law nonlinearity, (10) reads

$$
i D_{t}^{\alpha} q+a D_{t}^{\alpha} D_{x}^{\alpha} q+b D_{x}^{\alpha} D_{x}^{\alpha} q+c|q|^{2} q=0
$$

and Eq.(12) converts to

$$
(b-a v) U^{\prime \prime}-\left(w-a w \kappa+b \kappa^{2}\right) U+c U^{3}=0 .
$$

Comparing the highest order linear term $U^{\prime \prime}$ with nonlinear term $U^{3}$, we conclude that the $N=1$. This means that the series (7) must be expanded as the follows:

$$
U(\xi)=a_{0}+a_{1} \exp (-\phi(\xi))
$$

where $a_{0}$ and $a_{1}$ are constants to be determined, such that $a_{1} \neq 0$.

Plugging Eq.(15) into (14) by aid of Maple and putting the coefficients of same powers of $\exp (-\phi(\xi))$ to zero, we yield an algebraic systems of containing $a_{0}, a_{1}, \kappa, w, \lambda$ and $\mu$ as the follows:

$$
\begin{aligned}
-2 a v a_{1}+c a_{1}^{3}+2 b a_{1} & =0, \\
3 b a_{1} \lambda+3 c a_{0} a_{1}^{2}-3 a v a_{1} \lambda & =0, \\
-a v a_{1} \mu \lambda+a w \kappa a_{0}+b a_{1} \mu \lambda-w a_{0}+c a_{0}^{3}-b \kappa^{2} a_{0} & =0, \\
b a_{1} \lambda^{2}+a w \kappa a_{1}+3 c a_{0}^{2} a_{1}+2 b a_{1} \mu-2 a v a_{1} \mu-a v a_{1} \lambda^{2}-w a_{1}-b \kappa^{2} a_{1} & =0 .
\end{aligned}
$$


After solving system (16) through Eq.(11), we get the following coefficient results:

$$
\begin{gathered}
a_{0}= \pm \lambda \sqrt{\frac{-b}{2 a^{2} \kappa^{2} c-4 a c \kappa+4 a^{2} \mu c-a^{2} \lambda^{2} c+2 c}} \\
a_{1}= \pm 2 \sqrt{\frac{-b}{2 a^{2} \kappa^{2} c-4 a c \kappa+4 a^{2} \mu c-a^{2} \lambda^{2} c+2 c}} \\
w=\frac{b\left(2 \kappa^{3} a-2 \kappa^{2}+4 a \kappa \mu+4 \mu-a \kappa \lambda^{2}-\lambda^{2}\right)}{2 a^{2} \kappa^{2}-4 a \kappa+4 a^{2} \mu-a^{2} \lambda^{2}+2} .
\end{gathered}
$$

Therefore, the solution of Eq.(14) is

$$
U(\xi)= \pm \lambda \sqrt{\frac{-b}{2 a^{2} \kappa^{2} c-4 a c \kappa+4 a^{2} \mu c-a^{2} \lambda^{2} c+2 c}} \pm 2 \sqrt{\frac{-b}{2 a^{2} \kappa^{2} c-4 a c \kappa+4 a^{2} \mu c-a^{2} \lambda^{2} c+2 c}} \exp (-\phi(\xi))
$$

Let us now discuss the following cases:

Case 1. If $\lambda^{2}-4 \mu>0, \quad \mu \neq 0$,

$$
\begin{aligned}
U_{1}(\xi)= & \pm \lambda \sqrt{\frac{-b}{2 a^{2} \kappa^{2} c-4 a c \kappa+4 a^{2} \mu c-a^{2} \lambda^{2} c+2 c}} \\
& \pm 2 \sqrt{\frac{-b}{2 a^{2} \kappa^{2} c-4 a c \kappa+4 a^{2} \mu c-a^{2} \lambda^{2} c+2 c}} \frac{2 \mu}{\left[-\sqrt{\lambda^{2}-4 \mu} \tanh \left(\frac{\sqrt{\lambda^{2}-4 \mu}}{2}\left(\xi+C_{1}\right)\right)-\lambda\right]}, \\
U_{2}(\xi)= & \pm \sqrt{\frac{-b}{2 a^{2} \kappa^{2} c-4 a c \kappa+4 a^{2} \mu c-a^{2} \lambda^{2} c+2 c}} \\
& \pm 2 \sqrt{\frac{-b}{2 a^{2} \kappa^{2} c-4 a c \kappa+4 a^{2} \mu c-a^{2} \lambda^{2} c+2 c}} \frac{2 \mu}{\left[-\sqrt{\lambda^{2}-4 \mu} \operatorname{coth}\left(\frac{\sqrt{\lambda^{2}-4 \mu}}{2}\left(\xi+C_{1}\right)\right)-\lambda\right]} .
\end{aligned}
$$

Case 2. If $\lambda^{2}-4 \mu>0, \quad \mu=0$,

$$
\begin{aligned}
U_{3}(\xi)= & \pm \lambda \sqrt{\frac{-b}{2 a^{2} \kappa^{2} c-4 a c \kappa+4 a^{2} \mu c-a^{2} \lambda^{2} c+2 c}} \\
& \pm 2 \sqrt{\frac{-b}{2 a^{2} \kappa^{2} c-4 a c \kappa+4 a^{2} \mu c-a^{2} \lambda^{2} c+2 c}} \frac{\lambda}{\exp \left(\lambda\left(\xi+C_{1}\right)\right)-1}
\end{aligned}
$$

Case 3: If $\lambda^{2}-4 \mu=0, \quad \mu \neq 0, \quad \lambda \neq 0$,

$$
\begin{aligned}
U_{4}(\xi)= & \pm \lambda \sqrt{\frac{-b}{2 a^{2} \kappa^{2} c-4 a c \kappa+4 a^{2} \mu c-a^{2} \lambda^{2} c+2 c}} \\
& \pm 2 \sqrt{\frac{-b}{2 a^{2} \kappa^{2} c-4 a c \kappa+4 a^{2} \mu c-a^{2} \lambda^{2} c+2 c}} \frac{\lambda^{2}\left(\xi+C_{1}\right)}{2\left[\lambda\left(\xi+C_{1}\right)+2\right]}
\end{aligned}
$$


Case 4: If $\lambda^{2}-4 \mu<0$ and $\mu \neq 0$,

$$
\begin{aligned}
& U_{5}(\xi)= \pm \lambda \sqrt{\frac{-b}{2 a^{2} \kappa^{2} c-4 a c \kappa+4 a^{2} \mu c-a^{2} \lambda^{2} c+2 c}} \\
& \pm 2 \sqrt{\frac{-b}{2 a^{2} \kappa^{2} c-4 a c \kappa+4 a^{2} \mu c-a^{2} \lambda^{2} c+2 c}} \frac{2 \mu}{\left[\sqrt{4 \mu-\lambda^{2}} \tan \left(\frac{\sqrt{4 \mu-\lambda^{2}}}{2}\left(\xi+C_{1}\right)\right)-\lambda\right]}, \\
& U_{6}(\xi)= \pm \lambda \sqrt{\frac{-b}{2 a^{2} \kappa^{2} c-4 a c \kappa+4 a^{2} \mu c-a^{2} \lambda^{2} c+2 c}} \frac{-b}{\left[\sqrt{4 \mu-\lambda^{2}} \cot \left(\frac{\sqrt{4 \mu-\lambda^{2}}}{2}\left(\xi+C_{1}\right)\right)-\lambda\right]} .
\end{aligned}
$$

Then, the solutions of (13) corresponding to (20), (21) (22), (23), (24) and (25) are given by

$$
\begin{aligned}
& q_{1}(x, t)=U_{1}(\xi) e^{i\left(-\kappa \frac{x^{\alpha}}{\alpha}+w \frac{t^{\alpha}}{\alpha}+\theta\right)} \\
& q_{2}(x, t)=U_{2}(\xi) e^{i\left(-\kappa \frac{x^{\alpha}}{\alpha}+w \frac{t^{\alpha}}{\alpha}+\theta\right)} \\
& q_{3}(x, t)=U_{3}(\xi) e^{i\left(-\kappa \frac{x^{\alpha}}{\alpha}+w \frac{t^{\alpha}}{\alpha}+\theta\right)} \\
& q_{4}(x, t)=U_{4}(\xi) e^{i\left(-\kappa \frac{x^{\alpha}}{\alpha}+w \frac{t^{\alpha}}{\alpha}+\theta\right)} \\
& q_{5}(x, t)=U_{5}(\xi) e^{i\left(-\kappa \frac{x^{\alpha}}{\alpha}+w \frac{t^{\alpha}}{\alpha}+\theta\right)} \\
& q_{6}(x, t)=U_{6}(\xi) e^{i\left(-\kappa \frac{x^{\alpha}}{\alpha}+w \frac{t^{\alpha}}{\alpha}+\theta\right)}
\end{aligned}
$$

where the value of $w$ is fixed in Eq.(19). In the below figure (Figure 1), the kink shape soliton profile of the model equation (10) is observed for some special choices of parameters.

\section{Parabolic-law Nonlinearity}

This law arises in the nonlinear interaction between Langmuir waves and electrons. It describes the nonlinear interaction between the high frequency Langmuir waves and the ion acoustic waves by ponder-motive forces [27].

For parabolic-law nonlinearity, we consider $F(s)=\gamma_{s}+\beta s^{2}$ where $\gamma$ and $\beta$ are constants. Therefore, Eq.(10) takes the form

$$
i D_{t}^{\alpha} q+a D_{t}^{\alpha} D_{x}^{\alpha} q+b D_{x}^{\alpha} D_{x}^{\alpha} q+c\left(\gamma|q|^{2}+\beta|q|^{4}\right) q=0
$$

and Eq.(12) converts to

$$
(b-a v) U^{\prime \prime}-\left(w-a w \kappa+b \kappa^{2}\right) U+\gamma U^{3}+\beta U^{5}=0 .
$$




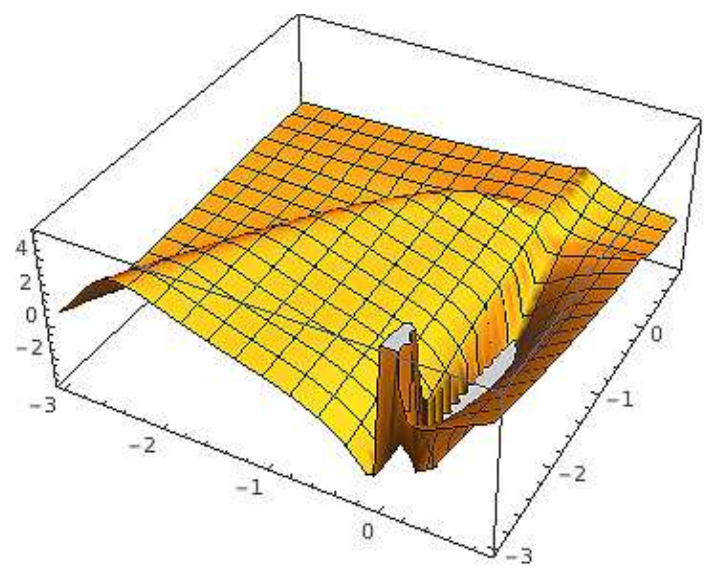

(a)

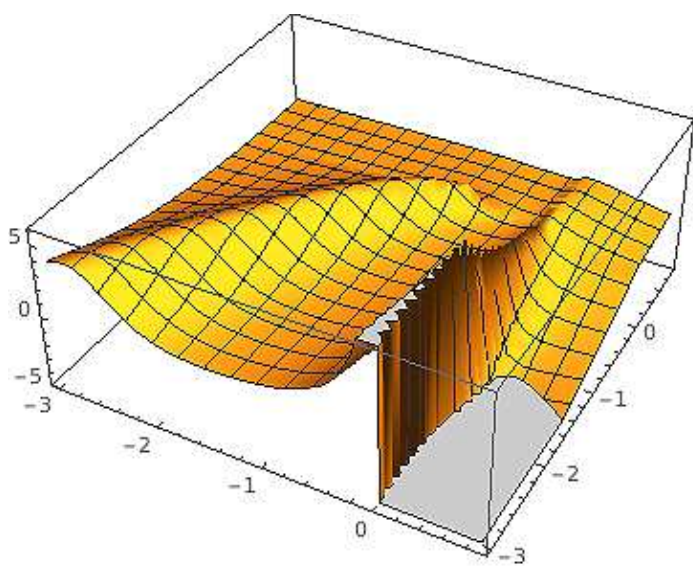

(b)

Fig. 1: The real and imaginary part of wave profile of Eq.(26) when $a=b=c=1, \kappa=1 / 2, C_{1}=0, w=2, \alpha=\frac{1}{2}, \theta=$ $1, \lambda=3, \mu=2$.

In order to use the balancing procedure, we need to make the ansatz $U=V^{\frac{1}{2}}$ in (33), so that (33) transforms to

$$
(b-a v)\left(2 V V^{\prime \prime}-V^{\prime 2}\right)-4 V^{2}\left(w-a w \kappa+b \kappa^{2}\right) U+4 \gamma V^{3}+4 \beta V^{4}=0 .
$$

Balancing $V V^{\prime \prime}$ with $V^{4}$ in Eq.(34) yields $N=1$. Thus, we have the following series expansion

$$
V(\xi)=a_{0}+a_{1} \exp (-\phi(\xi))
$$

Substituting $V$ and its necessary derivatives into (34) and equating all the coefficients of $\exp (-\phi(\xi)$ to zero, we obtain a highly complicated system of algebraic equations:

$$
\begin{aligned}
12 \gamma a_{0} a_{1}^{2}+24 \beta a_{0}^{2} a_{1}^{2}+2 b a_{1}^{2} \mu-4 b \kappa^{2} a_{1}^{2}+4 a w \kappa a_{1}^{2}+6 b a_{0} a_{1} \lambda-4 w a_{1}^{2}-2 a v a_{1}^{2} \mu+b a_{1}^{2} \lambda^{2}-6 a v a_{0} a_{1} \lambda-a v a_{1}^{2} \lambda^{2} & =0, \\
4 b a_{0} a_{1}+4 b a_{1}^{2} \lambda-4 a v a_{0} a_{1}+4 \gamma a_{1}^{3}-4 a v a_{1}^{2} \lambda+16 \beta a_{0} a_{1}^{3} & =0, \\
3 b a_{1}^{2}-3 a v a_{1}^{2}+4 \beta a_{1}^{4} & =0, \\
2 b a_{0} a_{1} \lambda^{2}+8 a w \kappa a_{0} a_{1}-8 w a_{0} a_{1}+4 b a_{0} a_{1} \mu+16 \beta a_{0}^{3} a_{1}-8 b \kappa^{2} a_{0} a_{1}-2 a v a_{0} a_{1} \lambda^{2}+12 \gamma a_{0}^{2} a_{1}-4 a v a_{0} a_{1} \mu & =0, \\
4 a w \kappa a_{0}^{2}+4 \gamma a_{0}^{3}+a v a_{1}^{2} \mu^{2}-4 b \kappa^{2} a_{0}^{2}-4 w a_{0}^{2}-2 a v a_{0} a_{1} \mu \lambda+4 \beta a_{0}^{4}+2 b a_{0} a_{1} \mu \lambda-b a_{1}^{2} \mu^{2} & =0 .
\end{aligned}
$$

Solving this system with the help of Maple, we reach to the following coefficient results through Eq.(11):

\section{Result 1:}

$$
\begin{aligned}
a_{0} & =\left(\frac{1}{2} \lambda+\frac{1}{2} \sqrt{\lambda^{2}-4 \mu}\right) a_{1} \\
\beta & =\frac{-3 b}{a_{1}^{2}\left(4 a^{2} \kappa^{2}-8 a \kappa+a^{2} \lambda^{2}-4 a^{2} \mu+4\right)}, \\
\gamma & =\frac{4 b}{a_{1}\left(4 a^{2} \kappa^{2}-8 a \kappa+a^{2} \lambda^{2}-4 a^{2} \mu+4\right)}, \\
w & =\frac{b\left(-4 \kappa^{2}+\lambda^{2}-4 \mu+4 \kappa^{3} a+a \kappa \lambda^{2}-4 a \kappa \mu\right)}{4 a^{2} \kappa^{2}-8 a \kappa+a^{2} \lambda^{2}-4 a^{2} \mu+4} .
\end{aligned}
$$


Then, the solutions of Eq.(32) corresponding to (35) are given by

$$
q_{1}(x, t)=\left[a_{0}+a_{1} e^{-\phi(\xi)}\right]^{\frac{1}{2}} e^{i\left(-\kappa \frac{x^{\alpha}}{\alpha}+w \frac{t^{\alpha}}{\alpha}+\theta\right)}
$$

where $\phi(\xi)$ holds Eq.(2.2) and $w$ is fixed in Eq.(35).

\section{Result 2:}

$$
\begin{aligned}
a_{0} & =\left(\frac{1}{2} \lambda-\frac{1}{2} \sqrt{\lambda^{2}-4 \mu}\right) a_{1} \\
\beta & =\frac{-3 b}{a_{1}^{2}\left(4 a^{2} \kappa^{2}-8 a \kappa+a^{2} \lambda^{2}-4 a^{2} \mu+4\right)}, \\
\gamma & =\frac{-4 b}{a_{1}\left(4 a^{2} \kappa^{2}-8 a \kappa+a^{2} \lambda^{2}-4 a^{2} \mu+4\right)}, \\
w & =\frac{b\left(-4 \kappa^{2}+\lambda^{2}-4 \mu+4 \kappa^{3} a+a \kappa \lambda^{2}-4 a \kappa \mu\right)}{4 a^{2} \kappa^{2}-8 a \kappa+a^{2} \lambda^{2}-4 a^{2} \mu+4} .
\end{aligned}
$$

Again, the solutions of Eq.(32) corresponding to (37) are given by

$$
q_{2}(x, t)=\left[a_{0}+a_{1} e^{-\phi(\xi)}\right]^{\frac{1}{2}} e^{i\left(-\kappa \frac{x^{\alpha}}{\alpha}+w \frac{t}{\alpha}+\theta\right)}
$$

where $\phi(\xi)$ holds Eq.(2.2) and $w$ is fixed in Eq.(37).

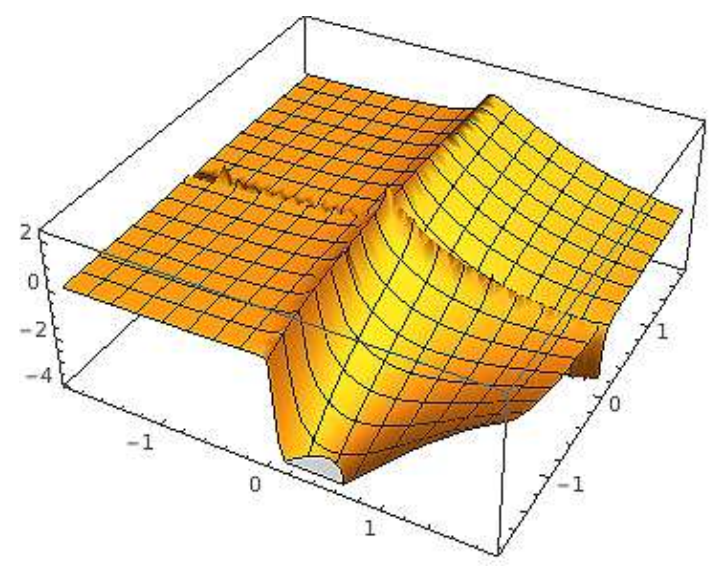

(a)

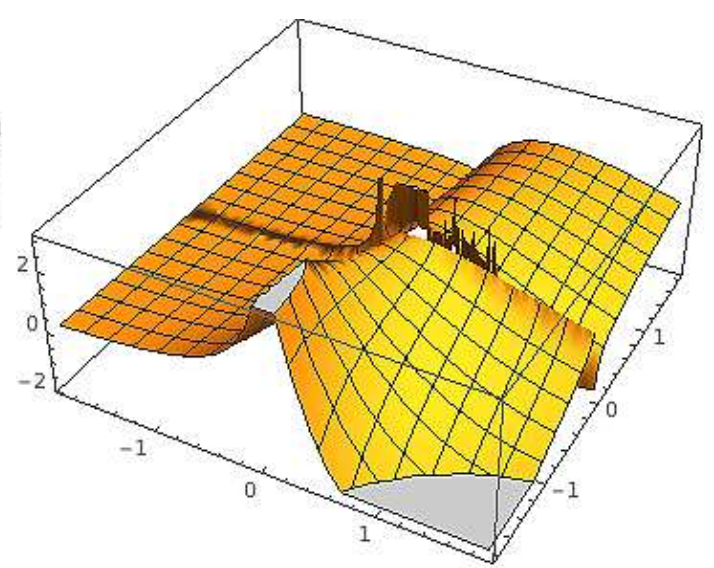

(b)

Fig. 2: The real and imaginary part of wave profile (multi-soliton shape) of Eq.(36) when $a=b=c=a_{1}=1, \kappa=\frac{1}{2}, C_{1}=$ $0, w=\frac{11}{10}, \alpha=\frac{1}{2}, \theta=1, \lambda=2, \mu=0$.

\section{Dual Power-law Nonlinearity}

Dual-power law nonlinearity is used to explain the saturation of the nonlinear refractive index. Moreover, this law serves as a basic model to describe the solitons in photovoltaic-photorefractive materials such as LiNbO3 [27]. The form of function $F(s)$ in this case is $F(s)=\gamma s^{n}+\beta s^{2 n}$ where $\gamma$ and $\beta$ are constants. Therefore, Eq.(10) takes the form

$$
i D_{t}^{\alpha} q+a D_{t}^{\alpha} D_{x}^{\alpha} q+b D_{x}^{\alpha} D_{x}^{\alpha} q+c\left(\gamma|q|^{2 n}+\beta|q|^{4 n}\right) q=0
$$


and Eq.(12) converts to

$$
(b-a v) U^{\prime \prime}-\left(w-a w \kappa+b \kappa^{2}\right) U+\gamma U^{2 n+1}+\beta U^{4 n+1}=0 .
$$

In order to use the balancing procedure, we need to make the ansatz $U=V^{\frac{1}{2 n}}$ so that Eq.(38) transforms to

$$
(b-a v)\left(2 n V V^{\prime \prime}+(1-2 n) V^{\prime 2}\right)-4 n^{2} V^{2}\left(w-a w \kappa+b \kappa^{2}\right) U+4 \gamma n^{2} V^{3}+4 \beta n^{2} V^{4}=0 .
$$

Balancing $V V^{\prime \prime}$ with $V^{4}$ in Eq.(39) yields $N=1$. Thus, we have the following series expansion

$$
V(\xi)=a_{0}+a_{1} \exp (-\phi(\xi))
$$

Substituting $V$ and its necessary derivatives into (39) and equating all the coefficients of $\exp (-\phi(\xi))$ to zero, we obtain a tedious the system of algebraic equations. Solving this system with the help of Maple, we reach to the following coefficient results through Eq.(11):

\section{Result 1:}

$$
\begin{aligned}
a_{0} & =\frac{\lambda}{2}+\frac{\sqrt{\lambda^{2}-4 \mu}}{2} a_{1}, \\
\beta & =\frac{-b(2 n+1)}{a_{1}^{2}\left(4 n^{2} a^{2} \kappa^{2}-8 n^{2} a \kappa-4 a^{2} \mu+a^{2} \lambda^{2}+4 n^{2}\right)}, \\
\gamma & =\frac{2 b\left(\sqrt{\lambda^{2}-4 \mu}-n \lambda+2 n\left(\frac{\lambda}{2}+\frac{\sqrt{\lambda^{2}-4 \mu}}{2}\right)\right)}{a_{1}\left(4 n^{2} a^{2} \kappa^{2}-8 n^{2} a \kappa-4 a^{2} \mu+a^{2} \lambda^{2}+4 n^{2}\right)}, \\
w & =\frac{b\left(4 n^{2} a \kappa^{3}-4 n^{2} \kappa^{2}+\lambda^{2}-4 a \mu \kappa-4 \mu+a \lambda^{2} \kappa\right)}{4 n^{2} a^{2} \kappa^{2}-8 n^{2} a \kappa-4 a^{2} \mu+a^{2} \lambda^{2}+4 n^{2}} .
\end{aligned}
$$

Then, the solutions of Eq.(39) corresponding to (40) are given by

$$
q_{1}(x, t)=\left[a_{0}+a_{1} e^{-\phi(\xi)}\right]^{\frac{1}{2}} e^{i\left(-\kappa \frac{x^{\alpha}}{\alpha}+w \frac{t^{\alpha}}{\alpha}+\theta\right)}
$$

where $\phi(\xi)$ holds Eq. (2.2) and $w$ is fixed in Eq.(40).

\section{Result 2:}

$$
\begin{aligned}
a_{0} & =\frac{\lambda}{2}-\frac{\sqrt{\lambda^{2}-4 \mu}}{2} a_{1}, \\
\beta & =\frac{-b(2 n+1)}{a_{1}^{2}\left(4 n^{2} a^{2} \kappa^{2}-8 n^{2} a \kappa-4 a^{2} \mu+a^{2} \lambda^{2}+4 n^{2}\right)}, \\
\gamma & =\frac{2 b\left(-\sqrt{\lambda^{2}-4 \mu}-n \lambda+2 n\left(\frac{\lambda}{2}-\frac{\sqrt{\lambda^{2}-4 \mu}}{2}\right)\right)}{a_{1}\left(4 n^{2} a^{2} \kappa^{2}-8 n^{2} a \kappa-4 a^{2} \mu+a^{2} \lambda^{2}+4 n^{2}\right)}, \\
w & =\frac{b\left(4 n^{2} a \kappa^{3}-4 n^{2} \kappa^{2}+\lambda^{2}-4 a \mu \kappa-4 \mu+a \lambda^{2} \kappa\right)}{4 n^{2} a^{2} \kappa^{2}-8 n^{2} a \kappa-4 a^{2} \mu+a^{2} \lambda^{2}+4 n^{2}} .
\end{aligned}
$$

Then, the solutions of Eq.(39) corresponding to (41) are given by

$$
q_{2}(x, t)=\left[a_{0}+a_{1} e^{-\phi(\xi)}\right]^{\frac{1}{2}} e^{i\left(-\kappa \frac{x^{\alpha}}{\alpha}+w \frac{t^{\alpha}}{\alpha}+\theta\right)}
$$

where $\phi(\xi)$ holds Eq.(2.2) and $w$ is fixed in Eq.(41). 


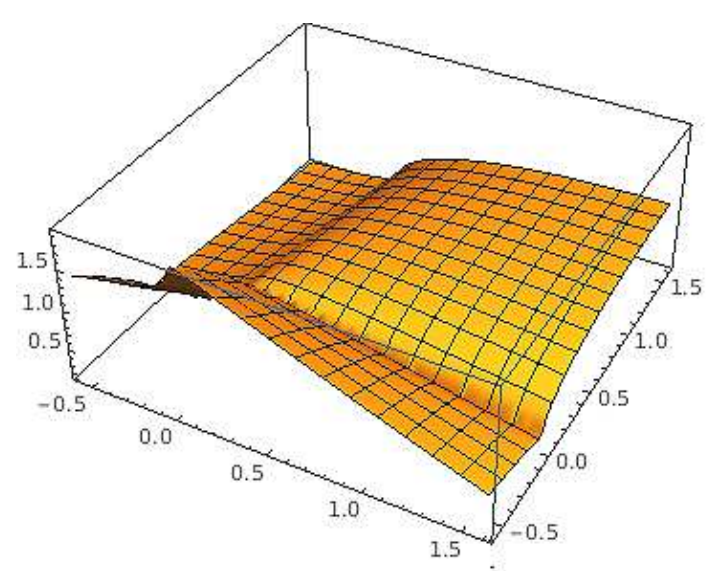

(a)

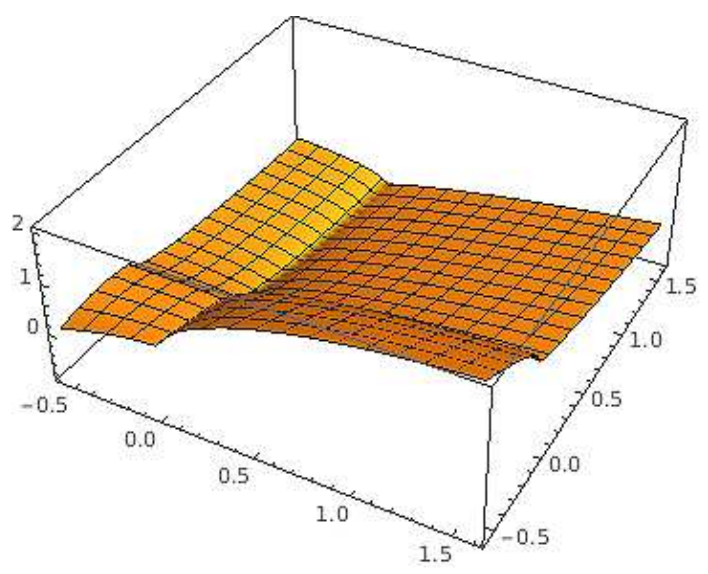

(b)

Fig. 3: The real and imaginary part of wave profile (kink shape) of Eq.(40) when $a=b=c=a_{1}=1, n=1, \kappa=\frac{1}{2}, C_{1}=$ $0, w=\frac{1}{2}, \alpha=\frac{1}{2}, \theta=0, \lambda=3, \mu=2$.

\section{Concluding remarks}

In this paper, we secured optical soliton solutions containing kink and multi-soliton shapes ( for instance, Eqs. (26), (36) and (40)) of space-time fractional NLSE with spatio-temporal dispersion. The model is examined in Kerr, parabolic and dual-power law cases separately. To best of our knowledge the physical model is examined for the first time in the form of conformal fractional derivative. The $\exp (-\phi(\xi))$ scheme was systematically used for revealing the solution sets of the under considered model. We note that due to the the balancing possibility we could use the series expansion. We shall focus the model equation together with the perturbation terms in the forthcoming works. The presented approach enables us to derive in a direct manner optical soliton solutions for the conformable fractional differential equations.

\section{Competing interests}

The authors declare that they have no competing interests.

\section{Authors' contributions}

All authors have contributed to all parts of the article. All authors read and approved the final manuscript.

\section{References}

[1] Arnous A.H., Mirzazadeh M., Zhou Q., Moshokoa S.P., Biswas A., Belic M., Optical solitons with higher order dispersions in parabolic law medium by trial solution approach, Optik-Int J Light Electron Opt, 127(23) (2016), 11306-11310.

[2] Bhrawy A.H., Alshaery A., Hilal E.M., Milovic D., Moraru L., Savescu M., Biswas A., Optical solitons with polynomial and triple power law nonlinearities and spatio-temporal dispersion, Proc. Rom. Acad. Ser. A, 15(3) (2014), 235-240.

[3] Hirota R., Exact solution of the Korteweg-de Vries equation for multiple collisions of solitons. Physical Review Letters, 27(18) (1971), 1192.

[4] Wang M., Zhou Y., Li Z., Application of a homogeneous balance methodto exact solutions of nonlinear equations in mathematical physics. Physics Letters A, 216 (1-5) (1996), 67-75. 
[5] He J.H., Wu X.H., Exp-function method for nonlinear wave equations. Chaos, Solitons \& Fractals, 30(3) (2006), 700-708.

[6] Eslami M., Vajargah B.F., Mirzazadeh, M., Biswas A., Application of first integral method to fractional partial differential equations. Indian Journal of Physics, 88(2) (2014), 177-184.

[7] Kudryashov N A., Simplest equation method to look for exact solutions of nonlinear dierential equations. Chaos, Solitons \& Fractals 24(5) (2005), 1217- 1231.

[8] Olver P.J., Applications of Lie groups to dierential equations (Vol. 107). Springer Science \& Business Media (2000).

[9] Wazwaz A.M., A sine-cosine method for handling nonlinear wave equations. Mathematical and Computer modelling, 40(5-6) (2004), 499-508.

[10] Lou S.Y., Hu X.B., Infinitely many Lax pairs and symmetry constraints of the KP equation. Journal of Mathematical Physics, 38(12) (1997), 6401-6427.

[11] Geng X., Lv Y., Darboux transformation for an integrable generalization of the nonlinear Schrödinger equation, Nonlinear Dynamics, 69 (2012), 1621-1630.

[12] Kumar S., Singh K., Gupta R.K., Coupled Higgs field equation and Hamiltonian amplitude equation: Lie classical approach and $G^{\prime} / G$ - expansion method, Pramana, 79 (2012), 41-60.

[13] Oldham K.B., Spanier J., The fractional calculus Academic Press, London (1974).

[14] Miller K.S., Ross B., An introduction to the fractional calculus and fractional differential equations Wiley, New York (1993).

[15] Samko S.G., Kilbas A.A., Marichev O.I., Fractional Integrals and Derivatives: Theory and Applications (Switzerland: Gordon and Breach Science Publishers) (1993).

[16] Podlubny I., Fractional differential equations: an introduction to fractional derivatives, fractional differential equations, to methods of their solution and some of their applications. Vol. 198. Elsevier, (1998).

[17] Caputo M., Linear Models of Dissipation Whose Q Is Almost Frequency Independent II. Geophysical Journal of the Royal Astronomical Society,13 (1967), 529-539.

[18] Jumarie G., Modified Riemann-Liouville derivative and fractional Taylor series of non-differentiable functions further results, Comput. Math. Appl., 51 (9-10) (2006), 1367-1376.

[19] Khalil R., Al-Horani M., Yousef A., Sababheh M., A new definition of fractional derivative, J. Comput. Appl. Math., 264 (2014), 65-70.

[20] Abdelrahman M.A.E., Zahran E.H.M., Khater M.M.A., Exact traveling wave solutions for power law and Kerr law non linearity using the $\exp (-\phi(\xi))$-expansion method, Global Journal of Science Frontier Research, 14(4) (2014).

[21] Khater M.M.A., Exact traveling wave solutions for the generalized Hirota-Satsuma couple KdV system using the $\exp (-\phi(\xi))$ expansion method, Cogent Mathematics, (2016) 3: 1172397

[22] Savescu M., Khan K.R., Naruka P., Jafari H., Moraru L., Biswas A., Optical solitons in photonic nanowaveguides with an improved nonlinear Schrödinger's equation, Journal of Computational and Theoretical Nanoscience, 10 (2013), 1182-1191.

[23] Yıldırım Y., Çelik N., Yaşar E., Nonlinear Schrödinger equations with spatio-temporal dispersion in Kerr, parabolic, power and dual power law media: A novel extended Kudryashov's algorithm and soliton solutions, Results in Physics, 7 (2017), 3116-3123.

[24] Biswas A., Mirzazadeh M., Eslami M., Zhou Q., Bhrawy A., Belic M., Optical solitons in nano-fibers with spatio-temporal dispersion by trial solution method, Optik-International Journal for Light and Electron Optics, 127(18) (2016), 7250-7257.

[25] Mohyud-Din S.T., Ali A., Exp(- $(\phi(\eta))$-expansion Method and Shifted Chebyshev Wavelets for Generalized Sawada-Kotera of Fractional Order, Fundam. Inform., 151 (2017), 173.

[26] Hosseini K., Mayeli P., Bekir A., Guner O., Density-Dependent Conformable Space-time Fractional Diffusion-Reaction Equation and Its Exact Solutions, Communications in Theoretical Physics, 69(1) (2018), 1.

[27] Biswas A., Fessak M., Johnson S., Beatrice S., Milovic D., Jovanoski Z., Optical soliton perturbation in non-Kerr law media: traveling wave solution, Opt LaserTechnol 44(1) (2012), 263-268. 